\title{
Diagnostic Accuracy of Radiologic Scoring System for Evaluation of Suspicious Hirschsprung Disease in Children
}

\author{
Mehdi Alehossein ${ }^{1,2}$; Ahad Roohi ${ }^{3}$; Masoud Pourgholami ${ }^{2}$; Mansour Mollaeian ${ }^{4}$; Payman \\ Salamati ${ }^{1,}$ \\ ${ }^{1}$ Advanced Diagnostic and Interventional Radiology Research Center (ADIR), Tehran University of Medical Sciences, Tehran, Iran \\ ${ }_{2}^{2}$ Department of Radiology, Bahrami Children Hospital, Tehran University of Medical Sciences, Tehran, Iran \\ ${ }^{3}$ Department of Radiology, Tehran University of Medical Sciences, Tehran, Iran \\ ${ }^{4}$ Department of Pediatric Surgery, Bahrami Children Hospital, Tehran University of Medical Sciences, Tehran, Iran \\ *Corresponding author: Payman Salamati, Advanced Diagnostic and Interventional Radiology Research Center (ADIR), Tehran University of Medical Sciences, Tehran, Iran. Tel: +98- \\ 2166581579, E-mail: psalamati@tums.ac.ir
}

Received: May 24, 2013; Revised: September 24, 2013; Accepted: October 3, 2013

\begin{abstract}
Background: In 1996, Donovan and colleagues represented a scoring system for better prediction of Hirschsprung disease(HD). Objectives: Our objective was to devise another scoring system that uses a checklist of radiologic and clinical signs to determine the probability of HD in suspicious patients.

Patients and Methods: In a diagnostic accuracy study, 55 children with clinical manifestations of HD that referred to a training hospital from 1998 to 2011 were assessed. A checklist was used to evaluate the items proposed by contrast enema (CE), based on six subscales, including transitional zone, rectosigmoid index (RSI), irregular contractions in aganglionic region, cobblestone appearance, filling defect due to fecaloid materials and lack of meconium defecation during the first 48 hours after birth. The patients were classified as high score and low score. Sensitivity, specificity, positive predictive value and negative predictive value of our scoring system were calculated for identifying HD, in comparison with pathologically proved or ruled out HD.

Results: Of the 55 patients, 36 (65.4\%) cases had HD and 19 (34.6\%) cases were without HD. In the HD group, 32 patients showed high scores and four patients had low scores. The sensitivity and specificity of our diagnostic scoring system were $88.9 \%$ (95\%CI:78.6\%-99.1\%) and $84.2 \%$ (95\% CI: 68.7\% - 100\%), respectively. Moreover, positive predictive value (PPV) and negative predictive value (NPV) were 91.4\% (95\% CI: $82.1 \%$ $100 \%$ and $80 \%(95 \%$ CI: 62.5\% - 97.5\%), respectively.

Conclusions: Our new scoring system of CE is a useful diagnostic method in HD. If a patient's score is high, that patient is highly suspicious to HD and reversely, when one's score is low, the patient presents a reduced probability to be diagnosed with HD.
\end{abstract}

Keywords: Hirschsprung Disease; Diagnosis; Sensitivity and Specificity

\section{Background}

Hirschsprung disease (HD) or congenital aganglionic megacolon is an intestinal motor disorder with an incidence of 1:5000 live births, ranging from 1:14000 to 1:4000 live births (1). It results in delayed meconium defecation, abdominal distension, lack of appetite, vomiting, and entrocolitis (2). In several cases, the patients may be asymptomatic until adolescence and puberty years and $15 \%$ of cases remain undiagnosed until the age of $5(2,3)$.

The gold standard of diagnosis is the pathological evaluation, revealing the absence of ganglion cells in the submucosal and myentric plexus, resulting in aperistaltism in the affected enteric regions and functional intestinal obstruction. Although the initial diagnosis is mainly based on clinical history and examination, followed only afterwards by pathological assessment (4), radiographic contrast assessment may be useful in diagnosis $(5,6)$. The risks associated with rectal biopsy, such as perfora- tion, scar, stricture, bleeding and also anesthesia-related adverse effects, results in seeking several non-invasive techniques, such as imaging methods $(7,8)$. A plain radiography may demonstrate a transition zone between a gas-filled colon and a non-dilated proximal colon, as a funnel-shaped region. Contrast enema (CE) with barium may be the first imaging procedure performed in the majority of centers, showing a transition zone, irregular colonic contractions, irregular mucosa suggesting enterocolitis, and an abnormal rectosigmoid index (RSI) (9). These methods may have different sensitivity and specificity, based on age and length of involved region (10-16). Regarding the availability and feasibility of CE in most centers, and considering the role of prompt diagnosis and treatment of disease for the prevention of misdiagnoses and complications, the use of a non-invasive method, such as CE, would help the physicians to achieve a better management of the disease. 


\section{Objectives}

In this study, a scoring system that was using a checklist of radiologic and clinical signs was developed and used for a more accurate diagnosis of HD, and the sensitivity and specificity were determined in comparison with biopsy, as a gold standard method.

\section{Patients and Methods}

In this cross-sectional study for determining the diagnostic accuracy of a radiologic checklist, the understudy population included 55 children with suspicious HD attending to a training referral hospital since 1998 to 2011, which undergone CE and HD diagnosis was ruled out or confirmed by full thickness bowel biopsy. Therefore, inclusion criteria were having clinical presentation highly suspicious for HD, performing a CE exam and full thickness biopsy. The study was approved by Ethical Committee of Tehran University of Medical Sciences, Tehran, Iran, and the Helsinki Declaration was respected entirely throughout the study.

Patients were divided into three age groups, as follows: $<1$ month, 1 - 12 months, $>12$ months. A checklist was used to evaluate the items proposed by $\mathrm{CE}$ as radiologic signs, including six subscales: 1) transitional zone(TZ), defined as significant change in intestinal diameter from non-dilated to dilated section during CE; 2) RSI, ratio of largest rectal diameter to largest sigmoid diameter (among proximal, distal, and loop sections), is considered abnormal if it is $<1$; 3) bizarre large irregular contractions in aganglionic region, with saw teeth appearance, due to dysrhythmia; 4) cobblestone appearance or mucosal irregularity or proximal colon spasm; 5) filling defect due to fecaloid materials; 6) lack of meconium defecation during the first 48 hours after birth. An example of cobble stone appearance and TZ is shown in Figure 1, while an example of bizarre irregular contraction is depicted by Figure 2. Figure 3 shows TZ and reversed RSI in a 3-month-old HD patient. For subscales 1 and 2 , if they were positive, we would consider scoring $=2$, and, if they were negative, we would consider scoring $=0$. For the other subscales, the positive results had scoring = 1 and negative results had score $=0$. Furthermore, the patients were evaluated based on scoring system, as follows: high (5-8), and low (0-4).

All the radiographies were interpreted by a radiologist without knowledge of the pathology results. Data analysis was performed with the SPSS version 20.0 software (IBM, USA). Chi-Square and independent $t$ tests were used for analysis and were considered statistically significant at $\mathrm{P}<0.05$.

\section{Results}

The mean age was $0.98 \pm 1.74$ months, ranging from 3 days to 8 years. Males represented $71 \%$ of patients while $29 \%$ were female. According to pathological results, 36 children (65.4\%) had HD and 19 subjects (34.6\%) had other diseases.
In HD group, 32 subjects showed high score (5-8) and four subjects low score (0-4). In the non-HD group, 16 subjects showed low score (0-4) and three subjects showed high score (5-8). The sensitivity and specificity of our diagnostic scoring system were 88.9\% (95\% CI: 78.6-99.1\%) and $84.2 \%$ (95\% CI: 68.7 - 100\%), respectively. Moreover, positive predictive value (PPV) and negative predictive value (NPV) were $91.4 \%$ (95\% CI: 82.1-100\%) and 80\% (95\% CI: 62.5-97.5\%), respectively.

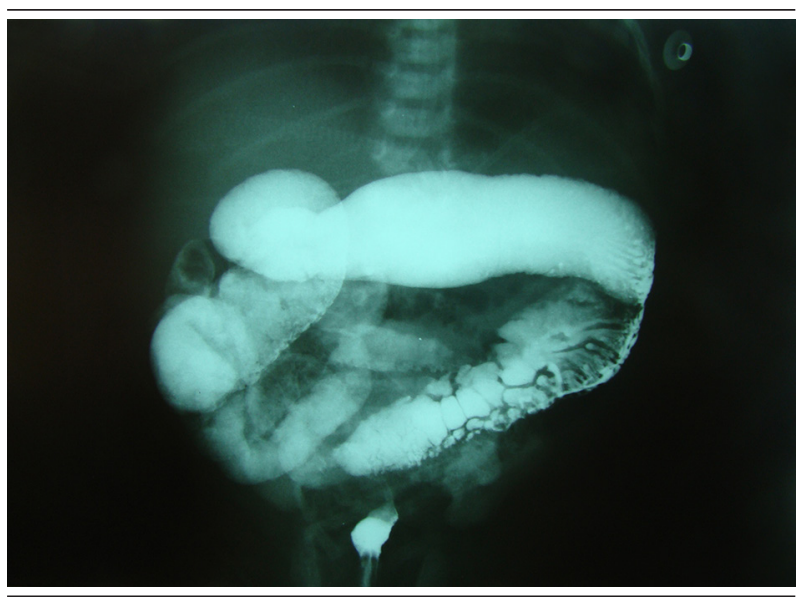

Figure 1. Barium enema in neonate with Hirschsprung disease shows transitional zone and cobblestone appearance

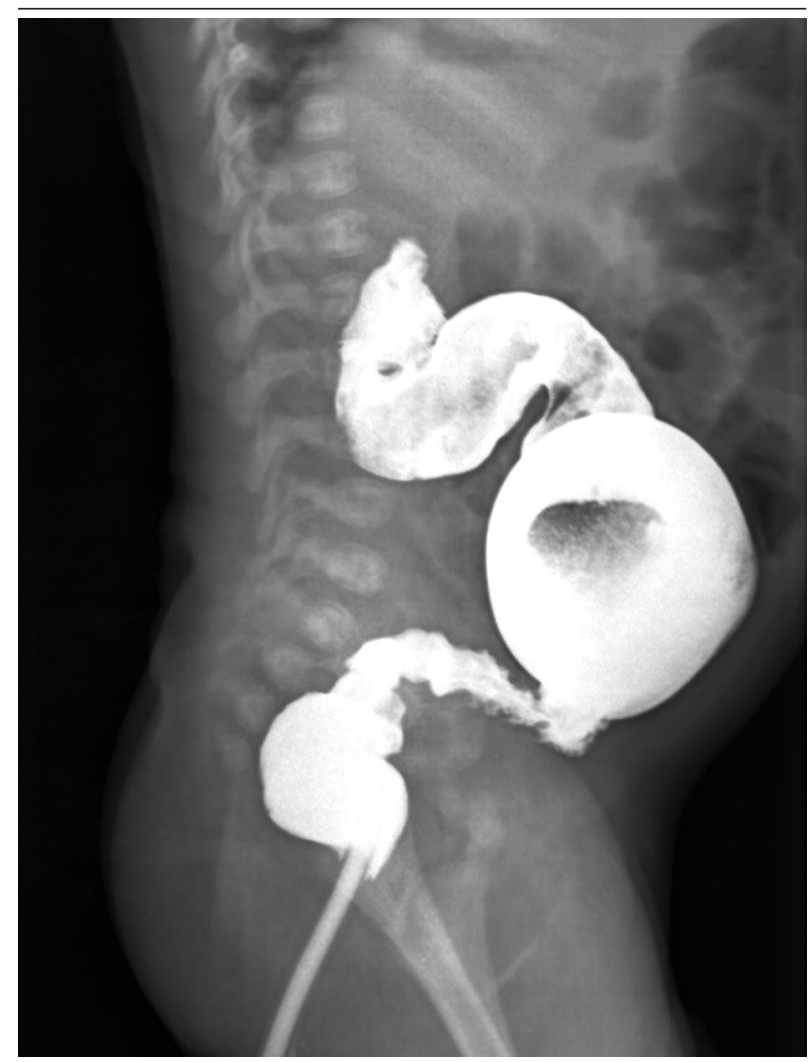

Figure 2. Barium enema in a 1-month-old male with Hirschsprung disease depicts bizarre irregular contraction 


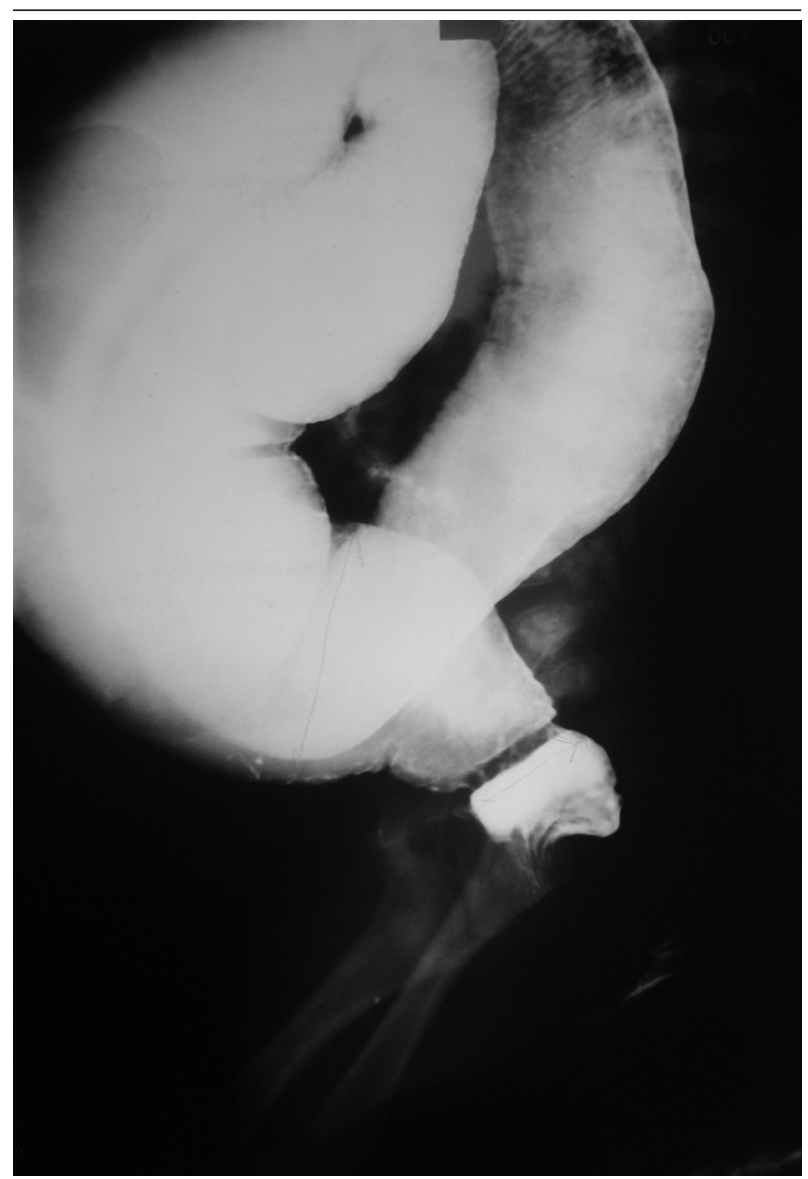

Figure 3. Barium enema shows transitional zone and reversed rectosigmoid index in a 3-month-old Hirschsprung disease patient
Abdominal distension, lack of meconium defecation, and constipation were the most common clinical symptoms with frequencies of $77.7 \%, 72.2 \%$, and $53 \%$, respectively. The most common findings in CE were TZ and RSI, respectively (Table 1). The sensitivity, specificity, PPV, NPV and accuracy for TZ in CE are shown in Table 2. These items are also demonstrated for each subscale by Table 3.

\section{Discussion}

Hirschsprung is an uncommon disease seen in all races and may result in decreased quality of life due to chronic constipation and periodic obstructions. It may also be fatal in severe cases with enterocolitis, functional obstruction, and intestinal perforation. Nowadays, the mortality has been decreased from $80 \%$ to $13 \%$, mainly owing to prompt diagnosis and subsequent conservative treatments (17), a trend that demonstrates the important role of definite correct diagnosis of HD.

Although the intestinal biopsy is the diagnostic gold standard, the first diagnostic method is CE (12, 18-20). The challenging issues of intestinal biopsy are higher cost, more invasiveness, longer hospital stay and need to follow-up $(18,21)$. Therefore, in this study, we compared our diagnostic scoring system with full thickness intestinal biopsy, as the gold standard diagnostic method.

In a similar study, Donovan et al. introduced a scoring system with eight items in CE, with scores 0 and 1 for negative and positive results, respectively and the patients were divided into three groups:

Table 1. Contrast Enema Findings in Those with and Without Hirschsprung a, b

\begin{tabular}{lccc}
\hline CE Findings & With Hirschsprung & Without Hirschsprung & PValue \\
\hline Transitional zone & $34(94)$ & $6(31.5)$ & 0.001 \\
Rectosigmoid index & $31(86)$ & $6(31.5)$ & 0.001 \\
Irregular contraction & $15(43)$ & - & - \\
Mucosal irregularity & $7(21)$ & - & - \\
Cobblestone appearance & $3(10.5)$ & $1(3.5)$ & $>0.05$ \\
Delay in passing meconium within 48 hours after birth & $26(72.2)$ & $5(26.3)$ & 0.001 \\
\hline
\end{tabular}

a Data are presented as No. (\%).

b Abbreviation: $\mathrm{CE}$, contrast enema.

Table 2. Diagnostic Indices of Transitional Zone ${ }^{a}$

\begin{tabular}{lcccc}
\hline Diagnostic Index & \multicolumn{3}{c}{ Time } \\
\cline { 2 - 5 } & $<$ Month & $\mathbf{1 - 1 2}$ Months & > 12 months & Total \\
\hline Sensitivity & 100 & 100 & 60 & 94.4 \\
Specificity & 42.8 & 80 & 85.7 & 68.4 \\
Positive predictive value & 81 & 93.3 & 75 & 85 \\
Negative predictive value & 100 & 100 & 75 & 86.7 \\
Accuracy & 89 & 90 & 75 & 85.4 \\
\hline
\end{tabular}

\footnotetext{
${ }^{a}$ Data are presented as $\%$.
} 
Alehossein $\mathrm{M}$ et al.

Table 3. Diagnostic Indices of Each Contrast Enema Findings a, b

\begin{tabular}{lcccccc}
\hline & TZ & RSI & Irregular Contraction & Cobblestone & Delay in Passing Meconium & Filling Defect \\
\hline Sensitivity & 94.4 & 86 & 41.6 & 18.3 & 72.2 & 61.1 \\
Specificity & 68.4 & 68.4 & 100 & 94.7 & 73.7 & 63.1 \\
Positive predictive value & 85 & 83.7 & 100 & 76.5 & 83.9 & 76 \\
Negative predictive value & 86.7 & 72.2 & 47.5 & 36.5 & 58.3 & 46.1 \\
Accuracy & 85.4 & 80 & 61.8 & 39.5 & 72.7 & 61.8 \\
\hline
\end{tabular}

${ }^{\mathrm{a}}$ Data are presented as \%.

b Abbreviations: RSI, rectosigmoid index; TZ, transition zone.

high (scores 6-8), moderate (scores 4-5), and low (scores 1-3) probability (22). However, this method of scoring was not very effective in our study and we decided to consider two points for positive results in RSI and TZ and one point for positive results related to lack of meconium defecation, cobblestone appearance, irregular contraction, and level of filling defect. We did not use delayed graphy and serration and also sex differences in our scoring system. In the current study, if one's score is high (5-8), the patient is highly suspicious to HD and if one's score is low (0-4), the patient presents a reduced probability to have HD, with high sensitivity and specificity. This demonstrates the helper role of scoring system in the diagnosis of HD. However, there are only limited other similar studies for the comparison of scoring systems.

Previous studies have shown the TZ and RSI as the most common signs in CE (23-25). The sensitivity and specificity of $\mathrm{CE}$ in the diagnosis of HD has shown a wide range, between $60 \%$ to $100 \%(24-26)$. This may be due to different techniques and also types of patient selections and different skill levels of the radiologists. Proctor et al. demonstrated that sensitivity and specificity of CE for short segment HD were $92 \%$ and $75 \%$, respectively, and they were $63 \%$ and $100 \%$ for long segment disease, respectively (15). Other factors such as age, sex, and disease duration may also be important in the prediction of sensitivity and specificity of CE in patients with older ages (23).

Reid et al. in their study, demonstrated that the NPV of $\mathrm{CE}$ in the post-neonatal period was higher than in the neonatal age and, oppositely, the PPV was high in each group. This shows the higher value of abnormal CE for HD confirmation compared with normal CE for rejection of HD diagnosis (27).

Similar to previous studies, the most common signs in CE were TZ and RSI. Among these, the TZ is pathognomonic for HD. CE would have both diagnostic and therapeutic efficacy in neonates. However, in absence of TZ, the HD may not be ruled out. In older children, the first differential diagnosis of HD is functional megacolon. In several review studies, the frequency of HD in those with TZ was reported at $72 \%$, while in the presence of other signs, it reduced to half (28).

Totally, according to obtained results, our diagnostic scoring system would have high validity for the diagnosis of HD and the scoring can be used as a useful diagnostic tool. However, further multi-centric studies, with larger sample sizes, are suggested for comparison of all diagnostic methods.

\section{Authors' Contributions}

The manuscript was resulted from Dr. Ahad Roohi's thesis. The supervisor professors of thesis were Dr. Mehdi Alehossein and Dr. Masoud Pourgholami. The advisors' professors of thesis were Dr. Payman Salamati and Dr. Mansour Mollaeian.

\section{References}

1. Rosai J. Rosai and Ackerman's Surgical Pathology. 9th edNew York: Mosby; 2004.

2. Kliegman RM, Behrman RE, Jenson HB, Stanton BF. Nelson Textbook of Pediatrics. 18th edPhiladelphia: Saunders Company; 2007.

3. Martucciello G. Hirschsprung's disease, one of the most difficult diagnoses in pediatric surgery: a review of the problems from clinical practice to the bench. Eur J Pediatr Surg. 2008;18(3):140-9.

4. Constipation Guideline Committee of the North American Society for Pediatric Gastroenterology H, Nutrition.. Evaluation and treatment of constipation in infants and children: recommendations of the North American Society for Pediatric Gastroenterology, Hepatology and Nutrition. J Pediatr Gastroenterol Nutr. 2006;43(3):e1-13.

5. Swenson O. Hirschsprung's disease: a review. Pediatrics. 2002;109(5):914-8.

6. Kapur RP. Practical pathology and genetics of Hirschsprung's disease. Semin Pediatr Surg. 2009;18(4):212-23.

7. Boman F, Corsois L, Paraf F. [Hirschsprung's disease: practical considerations]. Ann Pathol. 2004;24(6):486-98.

8. Wester T, Olsson Y, Olsen L. Expression of bcl-2 in enteric neurons in normal human bowel and Hirschsprung disease. Arch Pathol Lab Med.1999;123(12):1264-8.

9. de Lorijn F, Kremer LC, Reitsma JB, Benninga MA. Diagnostic tests in Hirschsprung disease: a systematic review. J Pediatr Gastroenterol Nutr. 2006;42(5):496-505.

10. De Lorijn F, Reitsma JB, Voskuijl WP, Aronson DC, Ten Kate FJ Smets AM, et al. Diagnosis of Hirschsprung's disease: a prospective, comparative accuracy study of common tests. J Pediatr. 2005;146(6):787-92.

11. Kim HJ, Kim AY, Lee CW, Yu CS, Kim JS, Kim PN, et al. Hirschsprung disease and hypoganglionosis in adults: radiologic findings and differentiation. Radiology. 2008;247(2):428-34

12. Diamond IR, Casadiego G, Traubici J, Langer JC, Wales PW. The contrast enema for Hirschsprung disease: predictors of a falsepositive result. J Pediatr Surg. 2007;42(5):792-5.

13. Garcia R, Arcement C, Hormaza L, Haymon ML, Ward K, Velasco $C$, et al. Use of the recto-sigmoid index to diagnose Hirschsprung's disease. Clin Pediatr (Phila). 2007;46(1):59-63.

14. Jamieson DH, Dundas SE, Belushi SA, Cooper M, Blair GK. Does 
Alehossein $M$ et al.

the transition zone reliably delineate aganglionic bowel in Hirschsprung's disease? Pediatr Radiol. 2004;34(10):811-5.

15. Proctor ML, Traubici J, Langer JC, Gibbs DL, Ein SH, Daneman A et al. Correlation between radiographic transition zone and level of aganglionosis in Hirschsprung's disease: Implications for surgical approach. J Pediatr Surg. 2003;38(5):775-8.

16. Pratap A, Gupta DK, Tiwari A, Sinha AK, Bhatta N, Singh SN, et al. Application of a plain abdominal radiograph transition zone (PARTZ) in Hirschsprung's disease. BMC Pediatr. 2007;7:5.

17. Khan AR, Vujanic GM, Huddart S. The constipated child: how likely is Hirschsprung's disease? Pediatr Surg Int. 2003;19(6):43942.

18. Kessmann J. Hirschsprung's disease: diagnosis and management. Am Fam Physician. 2006;74(8):1319-22.

19. Stranzinger E, DiPietro MA, Teitelbaum DH, Strouse PJ. Imaging of total colonic Hirschsprung disease. Pediatr Radiol. 2008;38(11):1162-70.

20. Lewis NA, Levitt MA, Zallen GS, Zafar MS, Iacono KL, Rossman JE, et al. Diagnosing Hirschsprung's disease: increasing the odds of a positive rectal biopsy result. J Pediatr Surg. 2003;38(3):412-6.

21. Ramanth N. Gastrointestinal Imaging. In: Blickman H editor Pediatr Radiol. 2nd ed: Elsevier; 2004.
22. O'Donovan AN, Habra G, Somers S, Malone DE, Rees A, Winthrop AL. Diagnosis of Hirschsprung's disease. AJR Am J Roentgenol. 1996;167(2):517-20.

23. Skaba R. Historic milestones of Hirschsprung's disease (commemorating the 90th anniversary of Professor Harald Hirschsprung's death). J Pediatr Surg. 2007;42(1):249-51.

24. Hebra A, Smith VA, Lesher AP. Robotic Swenson pull-through for Hirschsprung's disease in infants. Am Surg. 2011;77(7):937-41.

25. De la Torre-Mondragon L, Ortega-Salgado JA. Transanal endorectal pull-through for Hirschsprung's disease. J Pediatr Surg. 1998;33(8):1283-6.

26. Shankar KR, Losty PD, Lamont GL, Turnock RR, Jones MO, Lloyd DA, et al. Transanal endorectal coloanal surgery for Hirschsprung's disease: experience in two centers. $J$ Pediatr Surg. 2000;35(8):1209-13.

27. Reid JR, Buonomo C, Moreira C, Kozakevich H, Nurko SJ. The barium enema in constipation: comparison with rectal manometry and biopsy to exclude Hirschsprung's disease after the neonatal period. Pediatr Radiol. 2000;30(10):681-4.

28. Nasir AA, Adeniran JO, Abdur- Rahman LO. Hirschsprung's disease: 8 years experience in a Nigerian teaching hospital. J Indian Assoc Pediatr Surg. 2007;12(6):66-9. 